\title{
Labeled binary planar trees and quasi-Lie algebras
}

\author{
JEROME LEVINE
}

\begin{abstract}
We study the natural map $\eta$ between a group of binary planar trees whose leaves are labeled by elements of a free abelian group $H$ and a certain group $\mathrm{D}(H)$ derived from the free Lie algebra over $H$. Both of these groups arise in several different topological contexts. $\eta$ is known to be an isomorphism over $\mathbb{Q}$, but not over $\mathbb{Z}$. We determine its cokernel and attack the conjecture that it is injective.
\end{abstract}

$57 \mathrm{~N} 10 ; 57 \mathrm{M} 25$

This paper, Jerome Levine's fourth contribution to Algebraic and Geometric Topology, is published posthumously, following the author's untimely death in April 2006. The editors are very grateful to Kent Orr for preparing and proofreading the final version.

\section{Introduction}

Let $H$ be a finitely-generated free abelian group and $\mathrm{L}(H)$ the graded free Lie algebra on $H$. There is a natural homomorphism $H \otimes \mathrm{L}(H) \rightarrow \mathrm{L}(H)$ defined by bracketing, whose kernel is denoted $\mathrm{D}(H)$. If $H$ supports a non-singular symplectic form, eg $H=H_{1}(\Sigma)$, where $\Sigma$ is a closed orientable surface, with symplectic basis $\left\{x_{i}, y_{i}\right\}$, then $\mathrm{D}(H)$ is, in fact, a Lie algebra. It can be identified with the Lie subalgebra of $\mathcal{D}(\mathrm{L}(H)$ ) (the graded Lie algebra of derivations of $\mathrm{L}(H))$ consisting of those derivations which vanish on the element $\sum_{i}\left[x_{i}, y_{i}\right] \in \mathrm{L}_{2}(H)$.

$\mathrm{D}(H)$ has arisen in several different topological contexts. For example, it was probably first observed by Orr [14] (but see also Habegger and Lin [3]) that it is very natural to regard the Milnor invariants of a link $L$, or, more precisely, string link, as elements of $\mathrm{D}(H)$, where $H=H_{1}\left(S^{3}-L\right)$. If $\Sigma$ is a compact orientable surface with one boundary component and $H=H_{1}(\Sigma)$ then $\mathrm{D}(H)$ contains, as a Lie subalgebra, the associated graded Lie algebra of the relative weight filtration, defined by D. Johnson, of the mapping class group of $\Sigma-$ see Johnson [8] and Morita [13]. Similarly, if we consider the homology concordance group of homology cylinders over a surface - see Garoufalidis-Levine [2] and Levine [10] - there is also a relative weight filtration and, in this case, the associated graded Lie algebra is actually isomorphic to $\mathrm{D}(H)$. 
$\mathrm{D}(H)$ appears in Kontsevich's work [9] on graph complexes and his computation of the cohomology of the group of outer automorphisms of a free group.

Consider now the abelian group $\mathcal{A}^{t}(H)$ generated by unitrivalent trees, with cyclic orientations of its trivalent vertices and univalent vertices labelled by elements of $H$, subject to the anti-symmetry and IHX relations and linearity of the labels. $\mathcal{A}^{t}(H)$ appears as the indexing of the so-called tree-level of the Kontsevich integral of a link or string link. See Habegger and Masbaum [4] where this is related to the Milnor invariants via a natural map $\eta: \mathcal{A}^{t}(H) \rightarrow \mathrm{D}(H)$. It is proved there that, rationally, the Milnor invariants of a string link determine the tree-level of its Kontsevich integral. This corresponds to the fact that the map $\eta \otimes \mathbb{Q}: \mathcal{A}^{t}(H) \otimes \mathbb{Q} \rightarrow \mathrm{D}(H) \otimes \mathbb{Q}$ is an isomorphism, which is proved by Habegger and Pitsch [5] (see also Garoufalidis-Levine [2] and Levine [11]). $\mathcal{A}^{t}(H)$ appears in Habiro [6] and the study by Garoufalidis, Goussarov and Polyak [1] of claspers and finite-type invariants of 3-manifolds, and subsequently in Levine [10], mapping onto the associated graded groups of a filtration of the concordance group of homology cylinders defined using claspers. In this context the map $\eta: \mathcal{A}^{t}(H) \rightarrow \mathrm{D}(H)$ reflects the relation between the clasper filtration and the usual relative weight filtration. Most recently the group $\mathcal{A}^{t}(H)$ appears in the work of Schneiderman-Teichner [15], where it encodes the obstruction to removing intersection and self-intersection points of immersed connected surfaces in a simply-connected 4-manifold (here $H$ is a free abelian group of rank equal to the number of surfaces) via a tower of Whitney disks. In the special case where the surfaces are disks in the 4-ball bounded by a link in $S^{3}$, the obstruction element in $\mathcal{A}^{t}(H)$ maps to the element in $\mathrm{D}(H)$ corresponding to the Milnor invariants of the link.

In these various situations the study of the homomorphism $\mathcal{A}^{t}(H) \rightarrow \mathrm{D}(H)$ is closely related to the question of whether there are invariants in $\mathcal{A}^{t}(H)$ which give more information than the analogous, perhaps more easily defined, invariants in $\mathrm{D}(H)$. For example the work of Schneiderman-Teichner may uncover new invariants of link concordance beyond the Milnor invariants.

Since $\eta \otimes \mathbb{Q}$ is an isomorphism, the kernel and cokernel of $\eta$ are finite. In [11] some progress was made toward determining them. Toward this end we introduced the notion of a quasi-Lie algebra and studied the structure of a free quasi-Lie algebra. In the present work we extend these results. In particular we determine the precise structure of a free quasi-Lie algebra (adapting an argument of Marshall Hall [7]), determine the cokernel of $\eta$ precisely and show that a "good part" of the kernel of $\eta$ is trivial. It remains a reasonable conjecture that $\eta$ is injective.

It has recently come to our attention that some of the results of this note (and of [11]) have been independently obtained by K Habiro - in particular Theorem 2.1, Theorem 
4.1 in Section 4 (and Corollary 2.6) by a very similar method, as well as Theorem 2.7 by a different method.

The author was partially supported by an NSF grant.

\section{Statement of results}

We will use the precise definition of the groups $\left\{\mathrm{D}_{n}(H)\right\}$ and $\left\{\mathcal{A}_{n}^{t}(H)\right\}$ given in [10; 11]. In particular $\mathrm{D}_{n}(H)$ is the kernel of the bracket map $H \otimes \mathrm{L}_{n+1}(H) \rightarrow \mathrm{L}_{n+2}(H)$ and $\mathcal{A}_{n}^{t}(H)$ is a quotient of the free abelian group generated by unitrivalent trees with $n$ trivalent vertices, each of which is given an orientation - ie a cyclic ordering of its incident edges - and whose univalent vertices are labeled by elements of $H$. The "relations" which are divided out are:

- anti-symmetry: $T+T^{\prime}=0$, where $T^{\prime}$ is identical with $T$ except that one trivalent vertex is given the opposite orientation.

- IHX: $T_{1}-T_{2}+T_{3}=0$, where the $T_{i}$ are identical except in the neighborhood of two adjacent trivalent vertices, which look as follows:

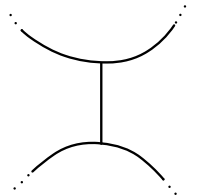

$T_{1}$

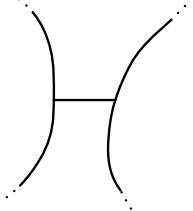

$T_{2}$

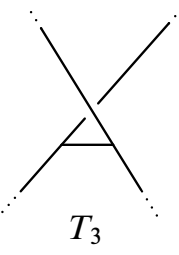

$T_{3}$

The orientations of the trivalent vertices are counterclockwise.

- linearity: $T=T_{1}+T_{2}$ where $T_{1}, T_{2}, T$ are identical except that one of the univalent vertices has labels $a_{1}, a_{2}, a_{1}+a_{2}$, respectively, for some $a_{i} \in H$.

The graphical representation of free Lie algebras over $\mathbb{Q}$, for example, is well-known. Extending this to Lie algebras over $\mathbb{Z}$ requires some extra considerations. In [11] we introduce the notion of a quasi-Lie algebra, in which the relation $[\alpha, \alpha]=0$ is replaced by the slightly weaker relation $[\alpha, \beta]+[\beta, \alpha]=0$. Then the free quasi-Lie algebra $\mathrm{L}^{\prime}(H)$ over a free abelian group $H$ is isomorphic to a Lie algebra of trees similar to the definition of $\mathcal{A}^{t}(H)$ except that one univalent vertex (the root) is not labeled.

There is an obvious epimorphism $\gamma_{n}: \mathrm{L}_{n}^{\prime}(H) \rightarrow \mathrm{L}_{n}(H)$. We can also define a bracketing homomorphism $\beta_{n}^{\prime}: H \otimes \mathrm{L}_{n+1}^{\prime}(H) \rightarrow \mathrm{L}_{n+2}^{\prime}(H)$ by $\beta_{n}^{\prime}(h \otimes \lambda)=[h, \lambda]$ and then define $\mathrm{D}_{n}(H)=\operatorname{Ker} \beta_{n}^{\prime}$. A map $\eta_{n}^{\prime}: \mathcal{A}_{n}^{t}(H) \rightarrow H \otimes \mathrm{L}_{n+1}^{\prime}(H)$ is defined by

$$
\eta_{n}^{\prime}(T)=\sum_{i} h_{i} \otimes\left[T_{i}\right]
$$


where the summation is over all univalent vertices of $T$. For each univalent vertex $h_{i}$ is its label and $T_{i}$ is the rooted tree obtained from $T$ by making that vertex the root $\left[T_{i}\right]$ is the corresponding element of $\mathrm{L}_{n+1}^{\prime}(H)$.

In [11] the following theorem is proved.

Theorem 2.1 The sequence

$$
\mathcal{A}_{n}^{t}(H) \stackrel{\eta_{n}^{\prime}}{\longrightarrow} H \otimes \mathrm{L}_{n+1}^{\prime}(H) \stackrel{\beta_{n}^{\prime}}{\longrightarrow} \mathrm{L}_{n+2}^{\prime}(H) \rightarrow 0
$$

is exact.

Therefore $\eta_{n}^{\prime}$ defines an epimorphism $\mathcal{A}_{n}^{t}(H) \rightarrow \mathrm{D}_{n}^{\prime}(H)$.

In order to completely understand the original map $\mathcal{A}_{n}^{t}(H) \rightarrow \mathrm{D}_{n}(H)$ we need to resolve the following two problems:

(1) Determine the map $\gamma_{n}: \mathrm{D}_{n}^{\prime}(H) \rightarrow \mathrm{D}_{n}(H)$, ie determine its kernel and cokernel.

(2) Determine the kernel of $\eta_{n}^{\prime}$.

Problem (1) essentially reduces to determining $\mathrm{L}_{n}^{\prime}(H)$, since $\mathrm{L}_{n}(H)$ is well-understood. In [11] it is shown that $\gamma_{n}$ is an isomorphism if $n$ is odd and, for $n$ even there is an exact sequence

$$
\mathrm{L}_{k}(H) \otimes \mathbb{Z} / 2 \stackrel{\theta_{k}}{\longrightarrow} \mathrm{L}_{2 k}^{\prime}(H) \stackrel{\gamma_{2 k}}{\longrightarrow} \mathrm{L}_{2 k}(H) \rightarrow 0
$$

where $\theta_{k}$ is defined by $\theta_{k}(\alpha)=[\alpha, \alpha]$ (where $\alpha \in \mathrm{L}_{k}(H)$ is lifted into $\mathrm{L}_{k}^{\prime}(H)$ ). It was conjectured in [11] that $\theta_{k}$ is injective. Our first result proves this conjecture.

Theorem 2.2 The sequence

$$
0 \rightarrow \mathrm{L}_{k}(H) \otimes \mathbb{Z} / 2 \stackrel{\theta_{k}}{\longrightarrow} \mathrm{L}_{2 k}^{\prime}(H) \stackrel{\gamma_{2 k}}{\longrightarrow} \mathrm{L}_{2 k}(H) \rightarrow 0
$$

is exact (and therefore split exact).

As a consequence of this theorem, we have the following relations between $\mathrm{D}_{n}^{\prime}(H)$ and $\mathrm{D}_{n}(H)$.

Corollary 2.3 There exist exact sequences:

$$
\begin{gathered}
0 \rightarrow \mathrm{D}_{2 k}^{\prime}(H) \rightarrow \mathrm{D}_{2 k}(H) \rightarrow \mathrm{L}_{k+1}(H) \otimes \mathbb{Z} / 2 \rightarrow 0 \\
0 \rightarrow H \otimes \mathrm{L}_{k}(H) \otimes \mathbb{Z} / 2 \rightarrow \mathrm{D}_{2 k-1}^{\prime}(H) \rightarrow \mathrm{D}_{2 k-1}(H) \rightarrow 0
\end{gathered}
$$


These exact sequences are derived in [11].

Remark 2.4 We can describe the elements of $\mathrm{D}_{2 k}(H)$ which do not come from $\mathrm{D}_{2 k}^{\prime}(H)$ in the following graphical manner. Let $\alpha \in \mathrm{L}_{k+1}(H)$ be represented by a labeled rooted tree $T$ of degree $k$. Let $T^{\prime}$ be another copy of $T$ and let $T \odot T^{\prime}$ be the labeled tree (representing an element of $\left.\mathcal{A}_{2 k}^{t}(H)\right)$ obtained by welding the roots of $T$ and $T^{\prime}$ together. For each labeled univalent vertex $v_{i}$ of $T$ let $T_{i} \odot T^{\prime}$ be the rooted labeled tree obtained from $T \odot T^{\prime}$ by making $v_{i}$ the root. If $h_{i}$ is the label of $v_{i}$ in $T$ then consider the element $\sum_{i} h_{i} \otimes\left(T_{i} \odot T^{\prime}\right) \in H \otimes \mathrm{L}_{2 k+1}^{\prime}(H)$. This does not lie in $\mathrm{D}_{2 k}^{\prime}(H)$ but its projection into $H \otimes \mathrm{L}_{2 k+1}(H)$ does lie in $\mathrm{D}_{2 k}(H)$ and maps to $\alpha \otimes 1 \in \mathrm{L}_{k+1}(H) \otimes \mathbb{Z} / 2$.

We now turn to Problem (2). In [11] the following is proved.

Theorem 2.5 $\eta_{n}^{\prime}$ is a split surjection. Ker $\eta_{n}^{\prime}$ is the torsion subgroup of $\mathcal{A}_{n}^{t}(H)$ if $n$ is even, and is the odd torsion subgroup of $\mathcal{A}_{n}^{t}(H)$ if $n$ is odd.

In both cases $(n+2) \operatorname{Ker} \eta_{n}^{\prime}=0$.

One immediate consequence is the known result that $\mathcal{A}_{n}^{t}(H) \otimes \mathbb{Q} \cong \mathrm{D}_{n}(H) \otimes \mathbb{Q}$.

We will improve on this result by constructing a splitting of $\mathcal{A}^{t}(H)$ and $\mathrm{D}^{\prime}(H)$ such that $\eta^{\prime}$ preserves components, and give a better estimate on the order of the kernel of each factor. In particular we will show:

Corollary 2.6 If $n+2$ is a prime power $p^{k}$, then $p^{k-1} \operatorname{Ker} \eta_{n}^{\prime}=0$. For example, if $n+2$ is prime, then $\eta_{n}^{\prime}$ is an isomorphism.

Finally, by a direct computation of ranks we will show:

Theorem $2.7 \quad \eta_{2}^{\prime}$ is an isomorphism.

Since it is obvious that $\eta_{1}^{\prime}$ is an isomorphism, the first unsettled case is $n=4$.

\section{Structure of the quasi-Lie algebra: Proof of Theorem 2.2}

Choose a basis $\left\{a_{1}, \ldots, a_{m}\right\}$ of $H$. Let $\mathcal{C}_{n}$ denote the set of formal commutators of degree $n$ in the $a_{i}$ and $\mathcal{C}=\cup_{n} \mathcal{C}_{n}$. Recall the definition of a Hall basis (see, for example, Hall [7]). Choose a linear ordering of the elements of $\mathcal{C}$ satisfying only that if $d(x)>d(y)$ (where $d$ denotes degree), then $x>y$. Let $\mathcal{H}$ be the subset of $\mathcal{C}$ defined recursively by the following properties: 
(1) Each $a_{i} \in \mathcal{H}$

(2) If $u, v \in \mathcal{C}$, then $[u, v] \in \mathcal{H}$ if and only if:

(a) $u, v \in \mathcal{H}$

(b) $u>v$

(c) If $u=[x, y]$ (and so $x, y \in \mathcal{H}$ and $x>y$ ), then $v \geq y$.

Note that $\mathcal{H}$ depends on the choice of ordering.

It is a well-known result (see, for example, [7]) that any Hall basis is a basis of the free Lie algebra $\mathrm{L}(H))$.

Let $\widetilde{\mathcal{H}}$ denote the subset of $\mathcal{C}$ consisting of all elements of the form $[h, h]$ for some $h \in \mathcal{H}$. It is clear that Theorem 2.2 will follow from:

Lemma 3.1 $L^{\prime}(H) \otimes \mathbb{Z} / 2$ has, as basis, $\mathcal{H}^{\prime}=\mathcal{H} \cup \tilde{\mathcal{H}}$.

Proof We will follow closely the proof in [7], making a few necessary modifications to apply to our situation.

Let $V_{n}$ be the $\mathbb{Z} / 2$-vector space with basis $\mathcal{C}_{n}, V=\oplus_{n} V_{n}$, and $W_{n}$ the $\mathbb{Z} / 2$-vector space with basis $\mathcal{H}_{n}^{\prime}$ and $W=\oplus_{n} W_{n}$. There are obvious maps:

$$
W_{n} \subseteq V_{n} \rightarrow \mathrm{L}_{n}^{\prime}(H)
$$

We will define a retraction $r: V_{n} \rightarrow W_{n}$ recursively on $n$, satisfying

(1) If $h \in \mathcal{H}^{\prime}$ then $r(h)=h$.

(2) For any $c \in \mathcal{C}, r(c)=c$ in $\mathrm{L}^{\prime}(H)$.

(3) For any $c_{1}, c_{2} \in \mathcal{C}, r\left[c_{1}, c_{2}\right]=r\left[r\left(c_{1}\right), r\left(c_{2}\right)\right]$.

For $n=1$ we define $r\left(a_{i}\right)=a_{i}$.

Now suppose $r$ is defined on $V_{k}$ for all $k<n$ satisfying (1)-(3). We will define a sequence of additive moves $V_{n} \rightarrow V_{n}$ which will define $r$ when it stops.

Step 1 If $c=\left[c_{1}, c_{2}\right]$, then $c \rightarrow\left[r\left(c_{1}\right), r\left(c_{2}\right)\right]$.

Now apply Step 2 to each term of the sum.

Step 2 If $c=\left[h_{1}, h_{2}\right]$, where $h_{1}, h_{2} \in \mathcal{H}^{\prime}$, then

$$
c \rightarrow \begin{cases}0 & \text { if } h_{1} \text { or } h_{2} \text { belongs to } \tilde{\mathcal{H}} \text { (case 1) } \\ c & \text { if } h_{1}, h_{2} \in \mathcal{H} \text { and } h_{1} \geq h_{2} \text { (case 2) } \\ {\left[h_{2}, h_{1}\right]} & \text { if } h_{1}, h_{2} \in \mathcal{H} \text { and } h_{1}<h_{2}(\text { case 3) }\end{cases}
$$

Algebraic 83 Geometric Topology, Volume 6 (2006) 
In case 1 stop. In case 2 or 3 go on to Step 3 .

Step 3 If $c=\left[h_{1}, h_{2}\right]$, with $h_{i} \in \mathcal{H}$ and $h_{1} \geq h_{2}$, write $h_{1}=\left[h_{3}, h_{4}\right]\left(\right.$ note $\left.h_{3}>h_{4}\right)$. Then

$$
c \rightarrow \begin{cases}c & \text { if } h_{2} \geq h_{4}(\text { case 1) } \\ {\left[\left[h_{3}, h_{2}\right], h_{4}\right]+\left[\left[h_{2}, h_{4}\right], h_{3}\right]} & \text { if } h_{2}<h_{4}(\text { case 2) }\end{cases}
$$

In case 1 stop. In case 2, apply Step 1 to each of the terms in the sum.

It is clear that if $c \in \mathcal{H}^{\prime}$, then the process will stop at Step 2 or 3 at $c$. In general we need to show that this process will stop after a finite number of steps. It is clear then that properties (1)-(3) will be satisfied.

Define a new relation among the elements of $\mathcal{C}_{n}$, for $n \geq 2$. Let $c=\left[c_{1}, c_{2}\right]$ and $c^{\prime}=\left[c_{1}^{\prime}, c_{2}^{\prime}\right]$. We will say

$$
c \succ c^{\prime} \text { if } \min \left(c_{1}, c_{2}\right)>\min \left(c_{1}^{\prime}, c_{2}^{\prime}\right)
$$

Now if $b$ is one of the terms in $\left[r\left(c_{1}\right), r\left(c_{2}\right)\right]$ obtained after Step 1, then applying Steps 2 and 3 to $b$ will either stop, resulting in an element of $\mathcal{H}^{\prime}$ or lead us to case 2 of Step 3. Take $\left[c_{1}^{\prime}, c_{2}^{\prime}\right]$ to be either of the resulting terms in case 2 of Step 3 . When we then apply Step 1 we have $\left[r\left(c_{1}^{\prime}\right), r\left(c_{2}^{\prime}\right)\right] \succ b$, since $r\left(c_{2}^{\prime}\right)=c_{2}^{\prime}>h_{2}$ and $d\left(r\left(c_{1}\right)\right)=d\left(c_{1}\right)>d\left(h_{2}\right)$. Thus iterating the process results in a sum of terms each of which stabilizes or leads to a sum of terms which are greater under the relation $\succ$. Since there are only a finite number of elements in $\mathcal{C}_{n}$ the process must eventually stop. In fact it must stop whenever the element $\left[h_{1}, h_{2}\right]$ to which we apply Step 3 satisfies $d\left(h_{2}\right)>\frac{n}{3}$ since this will force $d\left(h_{4}\right)<\frac{n}{3}$ from which it follows that $h_{2}>h_{4}$.

We now have defined a retraction $r: V_{n} \rightarrow W_{n}$ satisfying properties (1)-(3). To complete the proof we need to show that $r$ induces a map $L_{n}^{\prime}(H) \rightarrow W_{n}$. Notice that we can regard $V$ as the free $\mathbb{Z} / 2-$ magma over $H$ and that $L^{\prime}(H) \otimes \mathbb{Z} / 2$ is the quotient of $V$ by the ideal $I$ generated by elements of the form

$$
\xi=[x, y]+[y, x]] \quad \text { or } \quad[[x, y], z]+[[y, z], x]+[[z, x], y]
$$

where $x, y, z \in \mathcal{C}$. So we need to show that $r(I)=0$.

Now $I$ is generated additively by formal brackets of elements of $V$, one of which is of the form $\xi$ above. By property (3) of $r$ it is only necessary then to show that $r(\xi)=0$. In fact, again by property (3), we may assume that $x, y, z \in \mathcal{H}^{\prime}$.

$\xi=[x, y]+[y, x]:$ We may assume $x \geq y$. In the definition of $r$, we see that Step 2 will change $\xi$ either to 0 , if $x$ or $y$ belongs to $\tilde{\mathcal{H}}$, or to $2[x, y]=0$ otherwise. 
$\xi=[[x, y], z]+[[y, z], x]+[[z, x], y]:$ We may assume $x \geq y \geq z$ and proceed by induction on $d([[x, y], z])$.

Case $1 x=y$ : So $\xi=[[x, x], z]+[[x, z]+[z, x], x]$. Now $[x, x]$ either belongs to $\widetilde{\mathcal{H}}($ if $x \in \mathcal{H})$ or $r([x, x])=0$ if $x \in \widetilde{\mathcal{H}}$. In either case $r([[x, x], z])=0$.

For the remaining terms note that $[x, z]+[z, x] \rightarrow 2[x, z]=0$.

Case $2 x \in \tilde{\mathcal{H}}$ : Then $r([x, y])=r([[y, z], x])=r([z, x])=0$ by Step 2.

Case $3[x, y] \in \mathcal{H}$ : In evaluating $r([[x, y], z])$ we proceed to Step 3 and apply case 2:

$$
[[x, y], z] \rightarrow[[x, z], y]+[[z, y] x]
$$

At this point $\xi$ has been reduced to 0 .

Case $4[x, y] \notin \mathcal{H}^{\prime}$ and $x \in \mathcal{H}$ : We proceed by a downward lexicographical induction. Assume that $r(\xi)=0$ when

$$
\xi=\left[\left[x^{\prime}, y^{\prime}\right], z^{\prime}\right]+\left[\left[y^{\prime}, z^{\prime}\right], x^{\prime}\right]+\left[\left[z^{\prime}, x^{\prime}\right], y^{\prime}\right]
$$

and $x^{\prime} \geq y^{\prime} \geq z^{\prime}$ and either $z^{\prime}>z$ or $z^{\prime}=z$ and $y^{\prime}>y$.

Write $x=\left[x_{1}, x_{2}\right]$. Since $x \in \mathcal{H}$, then $x_{1}, x_{2} \in \mathcal{H}$ and $x_{1}>x_{2}$. Since $[x, y] \notin \mathcal{H}$, then $x_{2}>y$.

We therefore have:

$$
\begin{aligned}
r([[x, y], z]) & =r\left(\left[\left[\left[x_{1}, x_{2}\right], y\right], z\right]\right) \\
& \left.=r\left(\left[\left(\left[x_{1}, y\right], x_{2}\right]+\left[\left[x_{2}, y\right], x_{1}\right]\right), z\right]\right)
\end{aligned}
$$

by Step 3, case 2, since $x_{2}>y$, and Step 2, case 3, for the first term, since $x_{1}>y$.

We can apply our downward induction to both terms, since $\left[x_{1}, y\right]>x_{2}>y$ and both $\left[x_{2}, y\right]$ and $x_{1}$ are $>y$ (using Step 2 case 3 , if $x_{1}>\left[x_{2}, y\right]$ ) to obtain:

(2) $r([[x, y], z])=r\left(\left[\left[x_{2}, z\right],\left[x_{1}, y\right]\right]+\left[\left[\left[x_{1}, y\right], z\right], x_{2}\right]\right.$

$$
\left.+\left[\left[x_{1}, z\right],\left[x_{2}, y\right]\right]+\left[\left[\left[x_{2}, y\right], z\right], x_{1}\right]\right)
$$

By our ongoing induction on $\operatorname{deg}([[x, y], z])$ we have

$$
\begin{aligned}
& r\left(\left[\left[x_{1}, y\right], z\right]\right)=r\left(\left[\left[x_{1}, z\right], y\right]+\left[[y, z], x_{1}\right]\right) \\
& r\left(\left[\left[x_{2}, y\right], z\right]\right)=r\left(\left[\left[x_{2}, z\right], y\right]+\left[[y, z], x_{2}\right]\right)
\end{aligned}
$$


Substituting these equalities into equation (2) gives

$$
\begin{array}{r}
r([[x, y], z])=r\left(\left[\left[x_{2}, z\right],\left[x_{1}, y\right]\right]+\left[\left[\left[x_{1}, z\right], y\right], x_{2}\right]+\left[\left[[y, z], x_{1}\right], x_{2}\right]\right. \\
\left.+\left[\left[x_{1}, z\right],\left[x_{2}, y\right]\right]+\left[\left[\left[x_{2}, z\right], y\right], x_{1}\right]+\left[\left[[y, z], x_{2}\right], x_{1}\right]\right)
\end{array}
$$

Now we write

$$
\begin{aligned}
r([[z, x], y]) & =r\left(\left[\left[z,\left[x_{1}, x_{2}\right]\right], y\right]\right)=r\left(\left[\left[\left[x_{1}, x_{2}\right], z\right], y\right]\right) \\
& =r\left(\left[\left[\left[x_{1}, z\right], x_{2}\right], y\right]+\left[\left[\left[x_{2}, z\right], x_{1}\right], y\right]\right)
\end{aligned}
$$

using the Jacobi identity on elements of degree $<\operatorname{deg}([[x, y], z])$.

We can now use our downward induction on each of the two terms on the right to get:

$$
\begin{array}{r}
r([[z, x], y])=r\left(\left[\left[x_{1}, z\right],\left[x_{2}, y\right]\right]+\left[\left[\left[x_{1}, z\right], y\right], x_{2}\right]+\left[\left[x_{2}, z\right],\left[x_{1}, y\right]\right]\right. \\
\left.+\left[\left[\left[x_{2}, z\right], y\right], x_{1}\right]\right)
\end{array}
$$

We can now add equations (3) and (4), cancelling out many of the terms, to get

$$
\begin{aligned}
r([[x, y], z]+[[y, z], x]+[[z, x], y])=r\left(\left[\left[[y, z], x_{1}\right], x_{2}\right]\right. \\
+ \\
\left.+\left[\left[[y, z], x_{2}\right], x_{1}\right]+\left[[y, z],\left[x_{1}, x_{2}\right]\right]\right)
\end{aligned}
$$

If $[y, z]>x_{2}$, then our downward induction, applied to $\left[\left[[y, z], x_{1}\right], x_{2}\right]$, will tell us that $r\left(\left[\left[[y, z], x_{1}\right], x_{2}\right]+\left[\left[[y, z], x_{2}\right], x_{1}\right]+\left[[y, z],\left[x_{1}, x_{2}\right]\right]\right)=0$. (In case $x_{1}<[y, z]$, we use Step 2.) If $[y, z]<x_{2}$ then we apply downward induction on $\left[\left[x_{1}, x_{2}\right],[y, z]\right]$.

Finally note that, since $x_{1}>x_{2}>y>z$, we conclude that

$$
\operatorname{deg} z \leq \frac{1}{4} \operatorname{deg}([[x, y], z])=\frac{n}{4},
$$

which ensures the beginning of the induction.

This completes the proof of Lemma 3.1 and Theorem 2.2.

\section{Study of $\eta^{\prime}$}

\subsection{A splitting of $\mathcal{A}_{n}^{t}(H)$}

We now consider the maps $\eta_{n}^{\prime}: \mathcal{A}_{n}^{t}(H) \rightarrow \mathrm{D}_{n}^{\prime}(H)$. In [11] it is proved that

$$
(n+2) \operatorname{Ker} \eta_{n}^{\prime}=0 .
$$

We will construct a splitting of the various groups

$$
\mathcal{A}^{t}(H), \mathrm{L}^{\prime}(H), H \otimes \mathrm{L}^{\prime}(H)
$$


so that $\eta^{\prime}$ will respect the summands of these splittings and then give better estimates of the order of Ker $\eta^{\prime}$ on each summand. The splitting will depend on the choice of a basis $\mathcal{B}=\left\{\alpha_{1}, \ldots, \alpha_{d}\right\}$ of $H$. We adopt a slightly different, but equivalent, view of $\mathcal{A}^{t}(H)$ as generated by vertex-oriented unitrivalent trees with univalent vertices labeled by elements of $\mathcal{B}$, subject to the anti-symmetry and IHX relations (but now the linearity relation is not needed). Similarly $L^{\prime}(H)$ is generated by formal brackets in the elements of $\mathcal{B}$, subject to anti-symmetry and Jacobi relations. Thus $\mathrm{L}^{\prime}(H)$ is graphically described as generated by vertex-oriented unitrivalent trees with univalent vertices labeled by elements of $\mathcal{B}$ and one unlabeled univalent vertex chosen as a "root", subject to anti-symmetry and IHX.

Let $\omega=\left(n_{1}, \ldots, n_{d}\right)$ be a sequence of non-negative integers. We will say that a labeled vertex-oriented unitrivalent tree has signature $\omega$ if exactly $n_{i}$ of the vertices are labeled by $\alpha_{i}$. A formal bracket has signature $\omega$ if exactly $n_{i}$ of the entries in the bracket are $\alpha_{i}$. Notice that each anti-symmetry, IHX or Jacobi relation is defined by a sum of trees or brackets which all have the same signature.

We now define $\mathcal{A}_{\omega}^{t}(H)$ to be the abelian group generated by labeled vertex-oriented unitrivalent trees of signature $\omega$, subject to the anti-symmetry and IHX relations, and $\mathrm{L}_{\omega}^{\prime}(H)$ to be the group generated by brackets of signature $\omega$, subject to anti-symmetry and Jacobi relations. It is clear that

$$
\mathcal{A}_{n}^{t}(H)=\oplus_{\omega} \mathcal{A}_{\omega}^{t}(H) \quad \mathrm{L}_{n}^{\prime}(H)=\oplus_{\omega} \mathrm{L}_{\omega}^{\prime}(H)
$$

where the sums range over all $\omega$ with $\sum_{i} n_{i}=n+2$, for $\mathcal{A}_{n}^{t}(H)$ and with $\sum_{i} n_{i}=n$ for $\mathrm{L}_{n}^{\prime}(H)$. Note that the Lie bracket defines a pairing

$$
\mathrm{L}_{\omega}^{\prime}(H) \otimes \mathrm{L}_{\omega^{\prime}}^{\prime}(H) \rightarrow \mathrm{L}_{\omega+\omega^{\prime}}^{\prime}(H)
$$

where, if $\omega=\left(n_{1}, \ldots, n_{d}\right)$ and $\omega^{\prime}=\left(n_{1}^{\prime}, \ldots, n_{d}^{\prime}\right)$, then $\omega+\omega^{\prime}=\left(n_{1}+n_{1}^{\prime}, \ldots, n_{d}+\right.$ $\left.n_{d}^{\prime}\right)$.

The map $\eta_{n}^{\prime}: \mathcal{A}_{n}^{t}(H) \rightarrow H \otimes \mathrm{L}_{n+1}^{\prime}(H)$ is defined in equation (1). If the tree $T$ in that formula has signature $\omega=\left(n_{1}, \ldots, n_{d}\right)$, then each term on the right side will be of the form $\alpha_{j} \otimes\left[T_{i}\right]$, for some $j$, and where $\left[T_{i}\right] \in \mathrm{L}_{n+1}^{\prime}(H)$ has signature $\omega_{j}=\left(n_{1}, \ldots, n_{j}-1, \ldots, n_{d}\right)$. Therefore we can write

$$
\eta_{n}^{\prime}(T)=\sum_{j} \alpha_{j} \otimes \lambda_{j} \quad \text { where } \lambda_{j} \in \mathrm{L}_{\omega_{j}}^{\prime}(H)
$$

Now define $\left(H \otimes \mathrm{L}^{\prime}(H)\right)_{\omega}=\sum_{j} \alpha_{j} \otimes \mathrm{L}_{\omega_{j}}^{\prime}(H)$. It is clear that

$$
H \otimes \mathrm{L}_{n+1}^{\prime}(H)=\oplus_{\omega}\left(H \otimes \mathrm{L}^{\prime}(H)\right)_{\omega},
$$


where $\omega$ ranges over all $\omega$ with $\sum_{i} n_{i}=n+2$ and $\eta_{n}^{\prime}\left(\mathcal{A}_{\omega}^{t}(H)\right) \subseteq\left(H \otimes \mathrm{L}^{\prime}(H)\right)_{\omega}$. If $\omega=\left(n_{1}, \ldots, n_{d}\right)$ we define $\delta(\omega)=$ greatest common divisor of $n_{1}, \ldots, n_{d}$.

\section{Theorem 4.1}

$$
\delta(\omega)\left(\operatorname{Ker} \eta_{n}^{\prime} \mid \mathcal{A}_{\omega}^{t}(H)\right)=0
$$

Proof In the proof of Theorem 2.1 in [11] we use a map $\rho_{n}: H \otimes \mathrm{L}_{n+1}^{\prime}(H) \rightarrow \mathcal{A}_{n}^{t}(H)$ which sends any labeled tree with a root to the same tree, forgetting which vertex is the root. The observation that $\rho_{n} \circ \eta_{n}^{\prime}=$ multiplication by $n+2$ shows that $(n+2) \operatorname{Ker} \eta_{n}^{\prime}=$ 0 .

Now it is clear that $\rho_{n}\left(H \otimes \mathrm{L}^{\prime}(H)\right)_{\omega} \subseteq \mathcal{A}_{\omega}^{t}(H)$. But the restriction of $\rho_{n}$ to $(H \otimes$ $\left.\mathrm{L}^{\prime}(H)\right)_{\omega}$ can be decomposed into a sum of maps $\rho_{n}^{\omega, i}:\left(H \otimes \mathrm{L}^{\prime}(H)\right)_{\omega} \rightarrow \mathcal{A}_{\omega}^{t}(H)$ defined by

$$
\rho_{n}^{\omega, i}\left(\alpha_{j} \otimes \lambda\right)= \begin{cases}\rho_{n}\left(\alpha_{j} \otimes \lambda\right) & \text { if } i=j \\ 0 & \text { if } i \neq j\end{cases}
$$

It is clear that $\rho_{n}^{\omega, i} \circ \eta_{n}^{\prime} \mid \mathcal{A}_{\omega}^{t}(H)$ is just multiplication by $n_{i}$, and so

$$
n_{i} \operatorname{Ker} \eta_{n}^{\prime} \mid \mathcal{A}_{\omega}^{t}(H)=0
$$

for $i=1, \ldots, d$.

This completes the proof.

\subsection{Proof of Corollary 2.6}

We only need show that, for any $\omega=\left(n_{1}, \ldots, n_{d}\right)$ with $\sum_{i} n_{i}=p^{k}, \delta(\omega) \mid p^{k-1}$. Clearly $\delta(\omega) \mid p^{k}$, so suppose $\delta(\omega)=p^{k}$. This can only happen if some $n_{i}=p^{k}$ and the remaining $n_{j}=0$, which means that every tree $T$ in the generating set of $\mathcal{A}_{\omega}^{t}(H)$ has all its univalent vertices labeled by $\alpha_{i}$. Choose two univalent vertices which are each connected by an edge to the same trivalent vertex. Unless $n=1$ this trivalent vertex is connected by its third edge to another trivalent vertex. If we apply the IHX relation here, we see that $T=0$ in $\mathcal{A}^{t}(H)$.

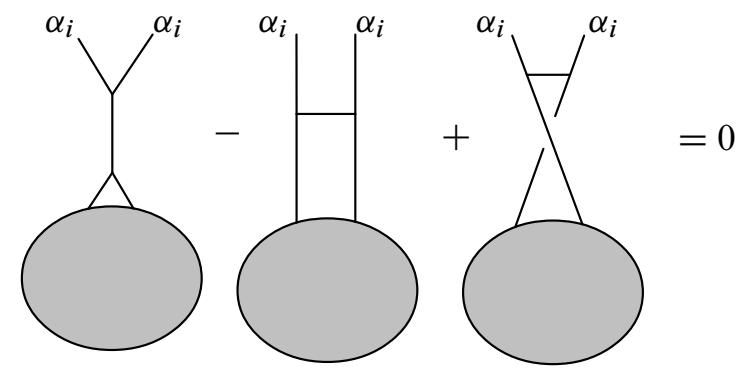

Algebraic $8 \mathcal{G}$ Geometric Topology, Volume 6 (2006) 
In case $n=1$, we see, by anti-symmetry, that $2 T=0$. But since $\delta(\omega)=3$ we also have $3 T=0$.

\subsection{Proof of Theorem 2.7}

If follows from Theorem 2.1 and Corollary 2.3 that $\mathrm{D}_{2}^{\prime}(H)=\operatorname{Im} \eta_{2}^{\prime}$ is a free abelian group of rank $d d_{3}-d_{4}$, where $d_{n}$ denotes the rank of $\mathrm{L}_{n}(H)$. Therefore in order to prove that $\eta_{2}^{\prime}$ is injective it suffices to show that $\mathcal{A}_{2}^{t}(H)$ is generated by $d d_{3}-d_{4}$ elements. Witt's formula (see, for example, Magnus, Karass and Solitar [12, Theorem 5.11]) gives a general formula for $d_{n}$ - in particular $d_{3}=2\left(\begin{array}{c}d+1 \\ 3\end{array}\right)$ and $d_{4}=\frac{1}{4}\left(d^{4}-d^{2}\right)$. Therefore $d d_{3}-d_{4}=\frac{1}{12}\left(d^{4}-d^{2}\right)$.

Now $\mathcal{A}_{2}^{t}(H)$ is generated by trees

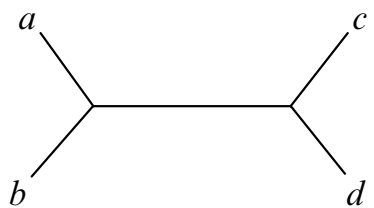

which we denote $T(a, b, c, d)$, where $a, b, c, d$ are elements of a basis $\mathcal{B}$ of $H$. Choose an ordering of $\mathcal{B}$.

Lemma 4.2 $\mathcal{A}_{2}^{t}(H)$ is generated by $\{T(a, b, c, d)\}$ with $a>b, c>d, a \geq c \geq b$ and, if $a=c, b \geq d$.

Assuming the lemma we can count the number of $T(a, b, c, d)$ satisfying the conditions of the lemma:

$$
\begin{aligned}
& \left(\begin{array}{l}
d \\
4
\end{array}\right) \quad \text { for } a>c>b>d \quad\left(\begin{array}{l}
d \\
4
\end{array}\right) \quad \text { for } a>c>d>b \\
& \left(\begin{array}{l}
d \\
3
\end{array}\right) \quad \text { for } a=c>b>d \quad\left(\begin{array}{l}
d \\
3
\end{array}\right) \quad \text { for } a>c=b>d \\
& \left(\begin{array}{l}
d \\
3
\end{array}\right) \quad \text { for } a>c>b=d \quad\left(\begin{array}{l}
d \\
2
\end{array}\right) \quad \text { for } a=c>b=d
\end{aligned}
$$

The sum of these six cases is exactly $\frac{1}{12}\left(d^{4}-d^{2}\right)$, which proves the Theorem.

Proof of Lemma 4.2 We first list some equalities:

$$
\begin{aligned}
& T(a, b, c, d)=T(d, c, b, a) \\
& T(a, b, c, d)=-T(b, a, c, d)=-T(a, b, d, c)=T(b, a, d, c) \\
& T(a, b, c, d)=T(a, c, b, d)-T(a, d, b, c) \\
& T(a, a, c, d)=T(a, b, c, c)=0
\end{aligned}
$$


Equation (6) follows by rotating the tree. Equation (7) is anti-symmetry and equation (8) is the IHX relation. Equation (9) follows from IHX (equation (8)) for $c=d$, and then from (6) for $a=b$.

We now prove the Lemma.

It follows from equations (7) and (9) that $\mathcal{A}_{2}^{t}(H)$ is generated by $T(a, b, c, d)$ with $a>b$ and $c>d$. Next let $\mathcal{T}$ consist of all $\{T(a, b, c, d)\}$ satisfying $a>b, c>d, a \geq c$ - we show that $\mathcal{T}$ generates $\mathcal{A}_{2}^{t}(H)$.

Suppose $a>b, c>d$ but $c>a$. Then we can use equations (6) and (7) to write $T(a, b, c, d)=T(c, d, a, b)$, which belong to $\mathcal{T}$.

Now define $\mathcal{T}^{\prime}$ to consist of all $\{T(a, b, c, d)\}$ satisfying $a>b, c>d, a \geq c \geq b$. Suppose $T(a, b, c, d) \in \mathcal{T}$ but $b>c$. Then apply equation (8) to $T(a, b, c, d)$ and note that $T(a, c, b, d)$ and $T(a, d, b, c)$ both belong to $\mathcal{T}^{\prime}$.

It remains to eliminate those $T(a, b, c, d) \in \mathcal{T}^{\prime}$ for which $a=c$ and $b<d$. Applying equations (7), (8) and (9) we have

$$
\begin{aligned}
T(a, b, a, d) & =T(a, a, b, d)-T(a, d, b, a) \\
& =T(a, d, a, b)
\end{aligned}
$$

This completes the proof.

\section{References}

[1] S Garoufalidis, M Goussarov, M Polyak, Calculus of clovers and finite type invariants of 3-manifolds, Geom. Topol. 5 (2001) 75-108 MR1812435

[2] S Garoufalidis, J Levine, Tree-level invariants of three-manifolds, from: "Proceedings of Stony Brook Conference in honor of Dennis Sullivan" (2005)

[3] N Habegger, X-S Lin, On link concordance and Milnor's $\bar{\mu}$ invariants, Bull. London Math. Soc. 30 (1998) 419-428 MR1620841

[4] N Habegger, G Masbaum, The Kontsevich integral and Milnor's invariants, Topology 39 (2000) 1253-1289 MR1783857

[5] N Habegger, W Pitsch, Tree level Lie algebra structures of perturbative invariants, J. Knot Theory Ramifications 12 (2003) 333-345 MR1983089

[6] K Habiro, Claspers and finite type invariants of links, Geom. Topol. 4 (2000) 1-83 MR1735632

[7] M Hall, Jr, A basis for free Lie rings and higher commutators in free groups, Proc. Amer. Math. Soc. 1 (1950) 575-581 MR0038336 
[8] D Johnson, A survey of the Torelli group, from: "Low-dimensional topology (San Francisco, Calif., 1981)”, Contemp. Math. 20, Amer. Math. Soc. (1983) 165-179 MR718141

[9] M Kontsevich, Formal (non)commutative symplectic geometry, from: "The Gel'fand Mathematical Seminars, 1990-1992”, Birkhäuser, Boston (1993) 173-187 MR1247289

[10] J Levine, Homology cylinders: an enlargement of the mapping class group, Algebr. Geom. Topol. 1 (2001) 243-270 MR1823501

[11] J Levine, Addendum and correction to: "Homology cylinders: an enlargement of the mapping class group”, Algebr. Geom. Topol. 2 (2002) 1197-1204 MR1943338

[12] W Magnus, A Karrass, D Solitar, Combinatorial group theory: Presentations of groups in terms of generators and relations, Interscience [John Wiley \& Sons] (1966) MR0207802

[13] S Morita, Abelian quotients of subgroups of the mapping class group of surfaces, Duke Math. J. 70 (1993) 699-726 MR1224104

[14] K E Orr, Homotopy invariants of links, Invent. Math. 95 (1989) 379-394 MR974908

[15] R Schneiderman, P Teichner, Whitney towers and the Kontsevich integral, from: "Proceedings of the Casson Fest”, Geom. Topol. Monogr. 7 (2004) 101-134 MR2172480

Department of Mathematics, Brandeis University

Waltham MA 02454-9110, USA

Correspondence to: K Orr

Department of Mathematics, Indiana University

831 East 3rd St, Bloomington IN 47405-7106, USA

korr@indiana.edu

http://php.indiana.edu/ korr/

Received: 23 December 2005 